
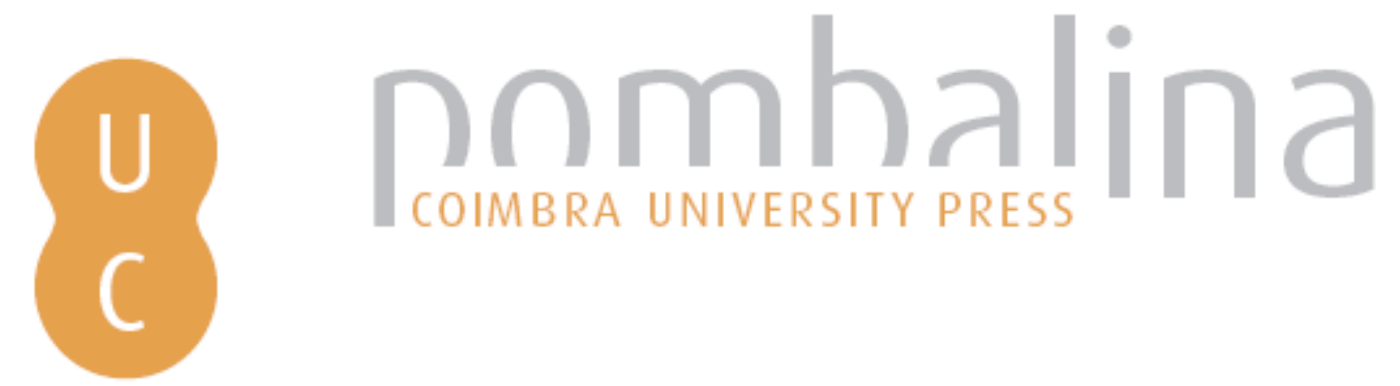

\title{
Una forma posible de interdisciplinariedad: la documentación iusfilosófica
}

Autor(es): $\quad$ Villaseñor Rodríguez, Isabel; Gómez García, Juan Antonio

Publicado por: Imprensa da Universidade de Coimbra

URL

persistente:

URI:http://hdl.handle.net/10316.2/31873

DOI:

DOI:http://dx.doi.org/10.14195/978-989-26-0319-3_12

Accessed : $\quad$ 26-Apr-2023 11:14:54

A navegação consulta e descarregamento dos títulos inseridos nas Bibliotecas Digitais UC Digitalis, UC Pombalina e UC Impactum, pressupõem a aceitação plena e sem reservas dos Termos e Condições de Uso destas Bibliotecas Digitais, disponíveis em https://digitalis.uc.pt/pt-pt/termos.

Conforme exposto nos referidos Termos e Condições de Uso, o descarregamento de títulos de acesso restrito requer uma licença válida de autorização devendo o utilizador aceder ao(s) documento(s) a partir de um endereço de IP da instituição detentora da supramencionada licença.

Ao utilizador é apenas permitido o descarregamento para uso pessoal, pelo que o emprego do(s) título(s) descarregado(s) para outro fim, designadamente comercial, carece de autorização do respetivo autor ou editor da obra.

Na medida em que todas as obras da UC Digitalis se encontram protegidas pelo Código do Direito de Autor e Direitos Conexos e demais legislação aplicável, toda a cópia, parcial ou total, deste documento, nos casos em que é legalmente admitida, deverá conter ou fazer-se acompanhar por este aviso. 
Maria Manuel Borges

Elias Sanz Casado

Coordenação

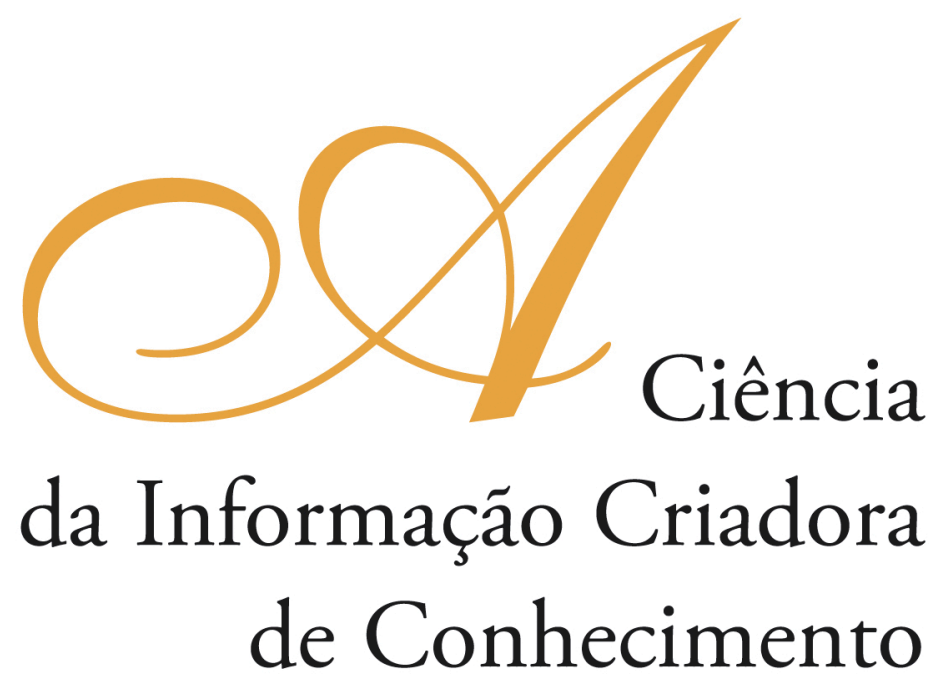

Vol. I

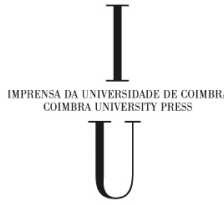

- COIMBRA 2009 


\title{
UNA FORMA POSIBLE DE INTERDISCIPLINARIEDAD: \\ La Documentación Iusfilosófica
}

\author{
Isabel Villaseñor Rodríguez \\ Universidad Complutense de Madrid (Espanha) \\ Juan Antonio Gómez García \\ Universidad Nacional de Educación a Distancia (Espanha)
}

\section{Resumen}

El trabajo presenta distintas consideraciones en torno a las relaciones que pueden existir entre dos disciplinas como son la Documentación y la Filosofía jurídica en el ámbito de los estudios universitarios. Tras el análisis del estatuto epistemológico de una y otra, se establecen las pautas necesarias para justificar su interrelación a partir de un caso práctico. Su objetivo es, pues, el de demostrar, en este contexto, la necesaria reciprocidad entre estas dos disciplinas y, en consecuencia, afirmar la posibilidad de estudios interdisciplinares tomando como referencia a ambas.

La metodología empleada en el presente trabajo se sustenta sobre el análisis de algunos textos filosófico-conceptuales, así como sobre la experiencia adquirida a partir de un estudio de la Documentación de carácter iusfilosófico y de sus usuarios en el ámbito académico.

Entre las conclusiones obtenidas se destaca la que se refiere a la necesidad de la interdisciplinariedad, no sólo en la forma de afrontar el tema desde un plano teórico-conceptual sino también en relación con sus estudiosos, esto es, los especialistas tanto en Documentación como en otras disciplinas, invitando a la necesaria cooperación entre unos y otros para indagar en lo que caracteriza a cada una de las disciplinas académicas desde el punto de vista de la Documentación. Asimismo, desde la perspectiva de la Filosofía jurídica, se toma conciencia de la necesaria formación de sus cultivadores en Documentación para un planteamiento y un desarrollo mejor fundados instrumentalmente de sus estudios.

\begin{abstract}
This paper presents considerations on the relations that can exist between two disciplines, such as the Documentation and the Philosophy of Law in the field of the university studies. After analyzing the epistemological status of both sets out the guidelines necessary to justify their interrelationships from a case study. Its aim is thus to demonstrate, in this context, the need for reciprocity between these two disciplines and, therefore, affirm the possibility of interdisciplinary studies with reference to both.

The methodology used in this paper is based on the analysis of philosophical texts and conceptual, as well as lessons learned from a study of the nature of the legal-philosophical Documentation and their users in Academia.

Among the findings highlighted that concerns the need for an interdisciplinary, not only on how to tackle the issue from a theoretical and conceptual level but also in relation to their studies, i.e. specialists in Documentation and in other disciplines, inviting the need for cooperation between them to find out what characterizes each of the academic disciplines
\end{abstract}


from the viewpoint of the Documentation. Also, from the perspective of Philosophy of law, is aware of the necessary training of farmers in their documentation and a better development of instrumentally based studies.

\section{Introducción: de la disciplina a la transdisciplinariedad}

La disciplina es una categoría que reviste un carácter evidentemente funcional desde un punto de vista epistemológico, en el sentido de constituir un criterio de organización del conocimiento científico. Su funcionalidad reside en su capacidad para instituir la división y la especialización del trabajo, al responder a la diversidad de los ámbitos que abarcan las ciencias. Aun perteneciendo a un espectro científico más amplio, cada disciplina tiende per se a la autonomía, a la delimitación de sus campos, y a la más depurada especificidad lingüística, de las técnicas que elabora y utiliza, y de su aparato teórico-conceptual propio.

La organización disciplinaria surge, tal y como la entendemos hoy, en el siglo XIX, al socaire de la formación de las universidades contemporáneas. Es en el siglo pasado cuando experimenta un desarrollo más intenso, a raíz del gran impulso de la investigación científica. Así pues, una razón de enorme peso en su conformación es la académica, en tanto que las instituciones académicas (especialmente las universidades) actúan como motores de primer orden en su origen, establecimiento, evolución, dispersión y desaparición. Por lo demás, son factores también sociológicos los que determinan estos procesos; sobre todo son la Sociología de las ciencias y la Sociología del conocimiento los otros elementos fundamentales en su configuración. La concurrencia de tales factores sociológicos demuestran en el fondo que no basta con ubicarse herméticamente en el interior de una disciplina cualquiera para conocer todas las cuestiones propias de ella, sino que debe producirse una apertura hacia el exterior para obtener una perspectiva más ajustada y profunda de las mismas, hasta el punto de que viene a ser una exigencia interna de la propia disciplina (Morin, ).

Cuando se pretende relacionar dos disciplinas de estudio, caben múltiples modos de vinculación que van desde su mera comparación horizontal (qué estudia cada una de ellas, qué métodos emplean para acercarse a su objeto, cuáles son sus procedimientos de trabajo propios, etc... al objeto de constatar sus diferencias) hasta su, podríamos decir, fusión en una única disciplina resultante de tal proceso. Ejemplo del primer tipo de aproximación sería la comparación entre el Derecho Civil y la Química Inorgánica, al objeto de comprender la muy diferente naturaleza epistemológica que tienen ambas disciplinas en tanto ámbitos científicos completamente distintos. El segundo tipo de aproximación podría ejemplificarse en el caso de la Política económica, consecuencia de la confusión en un solo campo entre la Política y la Economía. En este proceso, como puede comprobarse, caben distintas gradaciones que dependen de la mayor o menor cercanía epistemológica entre las disciplinas en cuestión, y del mayor o menor grado de acercamiento que se pretenda llevar a cabo entre ellas. Se hablaría ${ }^{1}$,

\footnotetext{
${ }^{1}$ Propuesta conceptual formulada por Erich Jantsch en el Seminario de la OCDE celebrado en 1979 (Cit. por León Hernández, 2009).
} 
en este sentido, de un amplio abanico que abarcaría desde lo que se viene llamando multidisciplinariedad, esto es, el grado más tenue de interrelación disciplinar, pasando por la pluridisciplinariedad, o simple yuxtaposición de disciplinas más o menos próximas en el contexto de un mismo ámbito de conocimiento, por la disciplinariedad cruzada, donde una disciplina domina sobre la otra u otras desde una relación de superioridad o de fuerza, por la propia interdisciplinariedad, que a continuación veremos, hasta llegar a la transdisciplinariedad, el más alto nivel de interdependencia hasta el punto de que se eliminan los límites entre las disciplinas y se llega a crear, como venimos diciendo, una nueva macrodisciplina, un nuevo modo de relación entre aquéllas, susceptible de ser empleada como modelo entre las distintas disciplinas.

Lo que resulta claro es que, en todos los supuestos posibles, lo que se está poniendo en ejercicio es una aproximación que aspira, de una forma u otra, a la mediación entre las disciplinas en cuestión. A nosotros nos interesa el tipo de mediación interdisciplinar, porque entendemos a priori que es la forma de interrelación disciplinar más realista, fructífera y viable entre los dos ámbitos concretos del conocimiento que pretendemos relacionar: la Filosofía jurídica y la Documentación. Además, de todos los acercamientos expuestos, la perspectiva interdisciplinar es, tal vez, la perspectiva epistemológica más interesante, más atractiva y más de moda en la actualidad por la variedad y riqueza de puntos de vista que admite. Precisamente la interdisciplinariedad constituye hoy una de las más provechosas aproximaciones metodológicas a explorar por parte del científico (singularmente en el caso del científico social por la propia flexibilidad metodológica que caracteriza a las llamadas Ciencias Sociales), ya que permite tematizar, de una manera eminentemente integradora, interactiva y horizontal, distintos aspectos de los campos científicos considerados, llegando a abrir (si se ejecuta bien) nuevas perspectivas, sin duda enriquecedoras y más acordes con las nuevas demandas científicas de los tiempos que corren, a menudo tan lamentablemente tendentes a la especialización cerrada, aislada y autorreferencial.

A pesar del carácter equívoco del concepto, evidentemente entendemos aquí por interdisciplinar toda aquella perspectiva científica que parte de la posibilidad de interrelaciones definidas (incluso de conexiones concretas y de integración) entre las disciplinas en cuestión, de sus teorías, métodos, técnicas, instrumentos y procedimientos de acción científica, con el fin de que sus actividades no se realicen de forma aislada, dispersa y fraccionada. Se parte como presupuestos, pues, de una concepción multidimensional de los fenómenos y del carácter relativo de los enfoques científicos por separado.

Como puede comprobarse, distinto es el concepto de transdisciplinariedad, el cual alude a la integración máxima de las disciplinas a partir de principios epistemológicos comunes, en un proceso lógico de carácter sintético, creándose así nuevos campos de conocimiento. Suele responder a una situación de extrema fragmentación del objeto científico de estudio, de tal modo que los procesos transdisciplinares vienen a componer (no decimos, re-componer, puesto que la transdisciplinariedad aboca a un estadio epistemológico distinto, ubicado más allá) objetos disueltos. En suma, como afirma M. Lopes Ginez de Lara (2009), “... la interdisciplinariedad moviliza el conocimiento de cada una de las disciplinas en diálogo, pretendiendo trascender la propia especialización aunque sin perder sus límites. No se trata de sumar, sino de interactuar para transformar. La interacción es, antes que nada, una actitud de comunicación, lo que permite 
verificar que, más que categoría de conocimiento, la interdisciplinariedad, así como la transdisciplinariedad, es una categoría de acción”(p.4).

Como decimos, en el presente trabajo vamos a ejercer una perspectiva interdisciplinar entre dos disciplinas científicas ${ }^{2}$ como son la Filosofía jurídica y la Documentación, con el propósito básico de afirmar la legitimidad de sus relaciones en el marco teóricopráctico de lo que denominamos como Documentación iusfilosófica, de explicitar éstas y de abrir posibilidades de ejercicio de posibles estudios interdisciplinares bajo este marco epistemológico.

\section{La Filosofía jurídica como disciplina}

La cuestión sobre el qué ha de entenderse bajo el rótulo Filosofía jurídica es de una extraordinaria complejidad. Se trata de un problema aún no resuelto a pesar de ser uno de los más antiguos y recurrentes de la reflexión filosófica occidental. Así pues, intentar ofrecer una respuesta más o menos definitiva puede resultar incluso osado; no obstante, tampoco puede renunciarse sin más a esta pretensión ya que la necesidad de acotar nuestro objeto de estudio impone la necesidad de llegar a una solución cuando menos funcional que nos permita caracterizarla, a los efectos de este trabajo, en un sentido meramente disciplinar.

Un sólido punto de partida que permite arrojar luz sobre este aspecto lo constituye la investigación histórica, ya que desde este punto de vista se gana en precisión a la hora de acotar la perspectiva y el ámbito temático de nuestra disciplina por su gran tradición. El enfoque histórico parte desde la consideración de la Filosofía jurídica como matriz disciplinar que contiene los diversos elementos que conforman un objeto de conocimiento homogéneo que ha experimentado un proceso de desarrollo temporal, el cual ha contribuido en muy alta medida a que en la actualidad sea lo que es como disciplina. Un esquema histórico que permite situar en sus justos términos la tarea de fijación de los contenidos concretos de ésta en la actualidad se dividiría en tres grandes fases temporales:

A) Una primera de dominio absoluto de la Metafísica: etapa de la doctrina del Derecho natural o iusnaturalismo. Desde un punto de vista general el iusnaturalismo es una forma de Filosofía jurídica que, históricamente, domina en su totalidad la reflexión iusfilosófica desde los comienzos de la Filosofía, en la Grecia clásica, hasta su crisis epistemológica derivada de la irrupción del modelo positivista en el siglo XIX. Dependiendo del pensador, escuela o época de que se trate, el iusnaturalismo aglutina multitud de tendencias y presenta gran diversidad de matices (Carpintero Benítez, 2000; Henkel, 1968; Márquez González, 1985; Recaséns Siches, 1970; Serrano Villafañe,

\footnotetext{
${ }^{2}$ Obviamente ha de entenderse aquí el término científico y el concepto de ciencia en los más amplios sentidos que quepan comprenderse: simple y llanamente como sinónimos de saber, de scientia en el sentido medieval escolástico del concepto. No entramos, pues, en reflexiones ni valoraciones en torno al estatuto epistemológico de estos saberes en relación con un determinado patrón de ciencia (especialmente el patrón empírico-naturalista moderno, como diría Kuhn, el paradigma de ciencia por excelencia), es decir, si con arreglo a tal patrón son auténtica ciencia o no, si sus métodos y sus resultados son propiamente científicos, si sus objetos son susceptibles de ser estudiados científicamente, etc...
} 
1977). Como afirma Villey (1984), bajo el término Derecho natural encontramos une cinquantaine de sens. No obstante, el iusnaturalismo mantiene una serie de características epistemológicas comunes que lo identifican como doctrina: básicamente el dualismo jurídico concretado en la distinción conceptual y metodológica entre Derecho natural y Derecho positivo, y la confusión esencial, en términos kantianos, entre ser y deber ser a la hora de entender el Derecho.

B) Una segunda caracterizada por el predominio del modelo científico fisicalista decimonónico: etapa del positivismo jurídico y, en palabras de F. González Vicén, de la Filosofía del Derecho en sentido estricto ${ }^{3}$. El contexto cultural en que surge está conformado por los siguientes elementos: el pujante historicismo, que da lugar al espectacular desarrollo de la moderna Ciencia de la Historia; el positivismo filosófico, para el que toda especulación que se sitúe más allá de los hechos constituye expresión de un estadio de desarrollo del espíritu humano ya superado; y el notable desenvolvimiento del saber teórico en torno al Derecho, que ha sufrido un importante proceso de división interna: por una parte, la Dogmática jurídica impulsada fuertemente por el movimiento codificador y que llega a alcanzar una específica pretensión de status de cientificidad (recuérdese, por ejemplo, la Escuela de la Exégesis), por otra, dentro del fenómeno genérico de desarrollo del saber histórico, la Historia del Derecho (la Escuela Histórica alemana) y el Derecho comparado adquieren un impulso decisivo con lo que ello supone de valorización del Derecho positivo como objeto propio de la Ciencia jurídica. En este panorama, lo verdaderamente importante es la preponderancia del modelo científicofisicalista como criterio excluyente de contraste, que plantea de inmediato la centralidad del problema de la ciencia y de la naturaleza científica de todo ámbito del saber que pretenda legitimarse como tal. La Filosofía no escapa a este planteamiento y surge por tanto la controversia referida al carácter científico de la Filosofía o, en su caso, a sus relaciones y conexiones. Esta situación tiene su correspondencia en la polémica entre la Filosofía jurídica y la Ciencia jurídica. La exclusión de la metafísica por constituir un pensamiento cuyos postulados no son verificables desde el punto de vista empírico o lógico-analítico, en un intento de ir más allá del trascendentalismo kantiano, tuvo como consecuencia el protagonismo absoluto de la teoría del conocimiento en los estudios filosóficos. De esta manera, para el positivismo decimonónico el conocimiento sólo puede versar sobre lo empíricamente mensurable, si bien enmarcándolo en una estructura lógica que da por supuesta la correspondencia entre los datos de la realidad empírica y los enunciados con que son descritos por nuestro lenguaje. En este sentido

\footnotetext{
${ }^{3}$ La expresión Rechtsphilosophie procede de Gustav Hugo, quien la utilizó por primera vez en su Lehrbuch eines civilistischen Cursus (1797). Antes de él los libros que se escribían sobre estas materias se adscribían a la expresión Derecho natural, con las excepciones de Conring, que hablaba de civilis philosophia, o de Kant, quien emplea la expresión Metaphysik der Sitten. Más tarde, Fries emplea el término philosophische Rechtslehre (en su obra del mismo título, 1803), Weiss habla de Philosophie des Rechtes (Lehrbuch der Philosophie des Rechtes, 1804) y Schmalz da en 1807 a la $4^{a}$ edición de su reines Naturrecht el título de Handbuch der Rechtsphilosophie. Estamos de acuerdo, pues, con González Vicén (1979a) cuando afirma: “... la Filosofía del Derecho no es una reflexión filosófica cualquiera sobre algo tan indeterminado llamado Derecho, sino una forma específica de reflexión caracterizada por el concepto también específico del Derecho que se halla en su base, y por referencia al cual cobra sentido. Frente a la escisión del horizonte jurídico en un Derecho postulado y un Derecho de la experiencia histórica, la Filosofía del Derecho se asienta sobre un monismo jurídico absoluto” (p. 215).
} 
la controversia sobre la relación entre la Filosofía y la Ciencia queda así resuelta: aquélla no es superior a ésta, sencillamente porque comparten el mismo objeto; la Filosofía no es otra cosa que Teoría de la Ciencia. Así se explica por qué la Filosofía del Derecho en sentido estricto se ceñirá exclusivamente al ámbito de la Ciencia jurídica rechazando cualquier cuestión valorativa como ajena a su objeto y enfoque, en aras de no perder así su carácter científico. En este paradigma son los hechos la piedra angular sobre la que se edifica toda la teoría del conocimiento ${ }^{4}$. El Derecho pasa a ser considerado como un fenómeno social con todas las consecuencias de esta caracterización: mutable, determinado, y cognoscible y explicable sólo por factores reales. Es el Derecho que es como hecho histórico-social, y no un supuesto Derecho ideal y trascendente, el objeto central de la Ciencia jurídica, desechándose por no científica cualquier otra tentativa de estudio de éste. Será, pues, la forma de lo jurídico, y no su contenido material, el objeto legítimo de conocimiento para una Filosofía del Derecho. En palabras de González Vicén, “... en tanto que conocimiento general del Derecho, la filosofía jurídica no puede ser conocimiento de los contenidos jurídicos, variables y condicionados, sino de su forma, es decir, de aquello que les presta su calidad de jurídicos. De esta manera (...) avanza al primer plano de la especulación el concepto formal del Derecho como Derecho positivo"(González Vicén, 1979b, p.129). En consecuencia, la Filosofía del Derecho no persigue una fundamentación metafísica del Derecho, su preocupación central es el concepto de Derecho. Así, junto a la investigación de los rasgos formales que lo distinguen de otros fenómenos sociales, se dedica también a la investigación de los presupuestos, carácter y procedimientos peculiares de la Ciencia jurídica, en definitiva del saber iusfilosófico específico surgido a raíz de la aparición del positivismo jurídico. Se esforzará, pues, por establecer los elementos estables y característicos del Derecho vigente, y por comprenderlo como el producto de procesos sociales presididos por relaciones lógico-causales, en cuya interrelación se explica lo jurídico.

C) Una tercera surgida de la crisis del positivismo y caracterizada por la diversidad de perspectivas metodológicas: etapa que llamaremos de pluralismo epistemológico. Durante las últimas décadas han sido numerosas las proclamaciones de crisis de la Filosofía del Derecho. Como producto del positivismo jurídico, debe entenderse (y así se ha manifestado en gran cantidad de ocasiones) que la crisis se extendió también en general a ésta. A semejante fenómeno van anejas las incisivas críticas formuladas contra la Filosofía en general, que han tenido como lugar común la proclamación de su muerte. Las causas de la crisis de la Filosofía del Derecho hay que buscarlas en las críticas vertidas contra el positivismo desde diversas posturas que, bien surgieron desde el propio positivismo en un intento de poner de manifiesto determinados aspectos que éste eludió o trató con demasiada poca atención y de criticar abiertamente al dominante positivismo legalista (en especial, su visión exclusivista de la ley como elemento fundamental para la identificación y la comprensión del Derecho, y su

\footnotetext{
${ }^{4}$ Así reza la regla fundamental del positivismo comtiano: “... toda proposición que no puede reducirse estrictamente al mero enunciado de un hecho, particular o general, no puede ofrecer ningún sentido real e inteligible. Los principios mismos que emplea no son ya más que verdaderos hechos, sólo que más generales y más abstractos que aquellos cuyo vínculo deben formar. Por otra parte, cualquiera que sea el modo, racional o experimental, de llegar a su descubrimiento, su eficacia científica resulta exclusivamente de su conformidad, directa o indirecta, con los fenómenos observados" (Comte, 1980, p. 28).
} 
concepto mecanicista de la aplicación de las normas jurídicas: los sociologismos, realismos, el llamado movimiento del Derecho libre, etc...), bien trataron de revitalizar el iusnaturalismo como teoría del Derecho bajo el lema el eterno retorno del Derecho natural (Rommen), sobre todo tras la II Guerra Mundial (doctrinas como, por ejemplo, la de die Natur der Sache de Radbruch, del Derecho Natural de contenido variable desde el neokantismo de Stammler, del Derecho Natural de contenido progresivo de Renard, o del Derecho Natural clásico de Villey), o bien significaron una crítica al positivismo desde la Filosofía actual rechazando su mentalidad cientificista, aunque también hay que decir que son producto de su discusión y en contraste con él (lo que Robles conceptúa bajo la denominación pospositivismo (Robles, 1988, p.107): la Hermenéutica, los Critical Legal Studies, el constructivismo jurídico, la Escuela de Frankfurt, etc...).

Desde la constatación de la diversidad de teorías expuestas no resulta difícil admitir que, en correspondencia con la tendencia filosófica general imperante, la discusión iusfilosófica presenta en la actualidad un panorama extraordinariamente complejo y plural, haciendo de su disciplina, la Filosofía jurídica, un ámbito extremadamente problemático y multiforme. En la conformación de tal diversidad, como ya se ha apuntado superficialmente con anterioridad, jugó un papel de primer orden la extendida consideración de la Filosofía fundamentalmente como Teoría de la Ciencia (Popper, 1965; Kuhn, 1971; Bunge, 1980). Esta concepción parece imponerse de manera pujante en el siglo XX, debido sobre todo al gran avance experimentado por las corrientes analíticas. En correspondencia con la reducción de la Filosofía a Lógica de la ciencia, se postula el abandono por parte de la Filosofía jurídica de los clásicos temas de Axiología y Ontología, ya que tratan sobre enunciados no verificables analítica ni empíricamente, y por tanto carentes de significado. Desde este presupuesto fundamental, el objeto de la Filosofía pasan a ser los problemas de carácter precientífico que la Ciencia da por supuestos y resueltos. En consonancia con esta situación, la Filosofía jurídica se convierte en reflexión teórica en torno a las Ciencias jurídicas, en una suerte de metaciencia jurídica. Se trata, en general, de resolver los problemas relacionados con la naturaleza y el status epistemológico del conocimiento jurídico, erigiéndose así la Epistemología en uno de los más importantes ámbitos de reflexión iusfilosófica.

No obstante, lo que viene a plantear actualmente en definitiva este pluralismo metódico al filósofo del Derecho como sujeto gnoseológico es el gran reto y la gran tarea de asumir las normas jurídicas (en una conexión de sentido con el resto de las reglas) como punto de partida sin caer en el normativismo excluyente, aceptar su referencia a valores evitando excesivas tentaciones metafísicas y, por último, relacionar todo el entramado normativo con los problemas sociológicos sin disolver lo jurídico en lo empírico-causal. Desde este equilibrio puede concebirse con justeza lo que pueda ser o formar parte de una Filosofía jurídica.

Parece, pues, evidente que la naturaleza de esta disciplina es eminentemente filosófica. Así pues, entendemos que la Filosofía jurídica actual, resultado del proceso histórico descrito, pretende integrar toda la temática y problemática que conlleva una reflexión metajurídica en torno al Derecho y le correspondería el desarrollo de un tipo de análisis que permita fundamentar, explicar criticamente y comprender la realidad jurídica, de lo que se sigue la necesidad de no limitarse al mero examen empírico del Derecho 
positivo, sino de extenderse a consideraciones de tipo filosófico con el fin de indagar en el sentido y función del Derecho en la vida humana y en la organización social.

Desde este tipo de análisis particular, un punto de acuerdo concreto en orden a la concreción de sus campos temáticos disciplinares es aquel que postula que la Filosofía jurídica estaría dividida en dos grandes bloques temáticos: uno, cuyo objeto sería el concepto y definición de Derecho y sus relaciones ontológicas con el resto de sistemas normativos y órdenes sociales; el otro, la Teoría General del Derecho, en cuyo ámbito se estudiaría la norma jurídica, el sistema jurídico y los conceptos jurídicos fundamentales. Como cierre estarían la teoría de la decisión y de la argumentación jurídicas, cuya función sería la de conectar los dos ámbitos anteriores, y el planteamiento de una Axiología jurídica o Teoría de los valores jurídicos con especial protagonismo de la Teoría de la justicia y de la Teoría de los derechos humanos, y el estudio de la metodología jurídica subyacente a todas ellas. Por lo tanto, el estudio de las relaciones entre lo jurídico y lo filosófico sería, de manera muy general, la razón de ser y la tarea de la disciplina iusfilosófica.

En suma, la Filosofía jurídica estudiaría todo aquello que atańe a la vastísima problemática que implica la consideración del Derecho como un producto cultural en el sentido más amplio de este concepto. He aquí el marco convencional entre los estudiosos de la disciplina que permite delimitar con rigor el amplísimo y muy problemático tema de qué deba entenderse bajo el rótulo Filosofía jurídica, y que a los efectos de la presente investigación resulta a todas luces suficiente para, desde un punto de vista funcional, establecer las bases de nuestra reflexión interdisciplinar con la Documentación.

\section{La Documentación como disciplina}

El término Documentación es un término que se puede considerar ambivalente ya que se utiliza para referirse tanto a una disciplina como a un conjunto de fuentes o recursos de información. En cuanto al primer sentido, esto es, como disciplina, cabe decir que, aunque existe una amplia bibliografía sobre el tema, podemos afirmar que se trata de una ciencia auxiliar de cualquier otra porque sistematiza las actividades desprendidas de la transmisión de conocimientos para organizarlos en fuentes con el fin de que sirvan para otros nuevos. De ahí su carácter de instrumento al servicio de las ciencias y su importancia en el progreso de las mismas, ya que favorece la producción y transmisión de todos los saberes así como la correcta transferencia de la información desde su creador hasta su usuario. Por otro lado, la Documentación se debe fundamentalmente al documento, entendido como fijación de la información, pero al documento estudiado desde un punto de vista especializado y aplicado a una ciencia determinada que transmite información científica, resultados. De ahí que se ocupe del crecimiento de la bibliografía científica, de la dispersión de las publicaciones periódicas, de la productividad de sus autores, de la correcta utilización de las fuentes que sirven de vehículo de transmisión de ideas científicas e, incluso, de las técnicas del trabajo científico. La Documentación es, según López Yepes, "la ciencia general que tiene por objeto el estudio del proceso de adecuación y transmisión de las fuentes para la obtención de nuevo conocimiento" (López Yepes, 1995, p. 322). Es la ciencia 
de las fuentes de información. Facilita al investigador las fuentes imprescindibles para obtener nuevo conocimiento científico.

En cuanto a la Documentación iusfilosófica podemos decir que cuenta con unas características peculiares derivadas de su especificidad propia (Villaseñor, 2009). Para empezar, debemos encuadrarla en lo que algunos autores han dado en llamar Documentación especializada en el sentido de que, como dice López Yepes, "se dirige al perfeccionamiento de los procesos de comunicación de la información científica en un campo del saber determinado" (López Yepes, 1995, p.323). La Documentación iusfilosófica es la Documentación aplicada a un saber en concreto para obtener nuevo conocimiento; es, pues, Documentación científica.

Es evidente que cada disciplina es particular con respecto a la documentación que genera y necesita. Las necesidades informativas de los usuarios de una disciplina serán distintas a las de los usuarios de otra con la que, incluso, tenga alguna relación. Están condicionadas por la actividad que desarrollen y por las características propias de cada uno de ellos (categoría académica, tipo de investigación, fuentes y recursos que use frecuentemente, por ejemplo). Esto va a determinar que cada disciplina requiera de un tipo de fuente que sea el más apropiado para su desarrollo e incluso que el proceso de búsqueda informativa, su estrategia, sea diferente al utilizado por otras. No sólo el tipo de fuente, sino también el orden que debe usarse para la búsqueda de la información requerida y la consulta de las fuentes puede (y así es) variar de un campo de saber a otro. Por lo tanto, es de crucial importancia afrontar el problema que para la Documentación especializada supone su organización, acceso y, fundamentalmente, su recuperación.

El estudioso de esa Documentación especializada debe, ante todo, conocer la estructura esencial de la disciplina de su interés así como las necesidades y comportamientos informativos de sus usuarios para luego poder aplicar la técnica documental necesaria que le permita conocer las fuentes pertinentes y su manejo. El proceso de identificación y caracterización de esas fuentes podría identificarse con lo que Pérez Álvarez-Osorio llama Documentación cientifica y que define como: "la actividad científica que se ocupa de la recogida y análisis de los documentos científicos y otras fuentes de nuevos conocimientos, almacenamiento de la información en ellos contenida, y recuperación y difusión de la misma, a fin de que alcance rápida y eficazmente a quienes puedan utilizarla” (Pérez Álvarez-Osorio, 1990, p.4). Romanos de Tiratel habla de fuentes de información especializadas como "suma de elementos disponibles que contienen un conjunto de símbolos con la capacidad de significar, dedicados a un área temática particular, registrados en cualquier soporte, con el potencial de poder recuperarse para satisfacer una necesidad del usuario..." (Romanos de Tiratel, 2000, p.25).

Dentro de esta condición de Documentación especializada, el tipo de Documentación que nos ocupa podría encuadrarse en la ya clásica categoría de Documentación en Humanidades y Ciencias Sociales por presentar características que son propias de unas y otras porque, al tener por objeto de estudio los comportamientos jurídicos y administrativos en tanto que hechos sociales que se generan en las diferentes esferas de la actividad social, puede considerarse como una Ciencia Social pero al considerar la Historia de esos comportamientos así como el pensamiento filosófico que los genera o se deriva de ellos (Filosofía) se encuadra también en el grupo de las Humanidades. 
Descendiendo aún más en el nivel de especificidad conceptual, la Documentación iusfilosófica es un tipo de Documentación jurídica, entendida esta última como "la generada en la creación, aplicación, difusión e investigación del Derecho. Abarca, por tanto, todas las fuentes del conocimiento del Derecho, esto es, la legislación, la jurisprudencia y la doctrina científica en cualquiera de sus soportes: papel o informático" (Manual de documentación juridica, 1998, p. 20). El conjunto de fijaciones de "todo conocimiento susceptible de producir efectos de una cierta relevancia en el campo de las Ciencias Jurídicas que sea tratado de forma adecuada para facilitar su transmisión mediante los correspondientes procesos informativos, permitiendo a una pluralidad de sujetos el acceso y utilización del mismo" (Páez Mañá, 1994, p.3) en unos soportes que permitan su inmutabilidad durante un tiempo, su transmisión y su recuperación, constituyen el núcleo fundamental de la Documentación jurídica, que transmite un saber entorno a un objeto concreto que es el Derecho.

Por último, la Documentación iusfilosófica, dentro de la clasificación establecida por autores como Reviriego y Maciá (Manual de documentación jurídica, 1998) para la Documentación jurídica, distinguiendo entre Documentación legislativa, parlamentaria, administrativa, judicial y de doctrina jurídica o científica, se encuadraría en la categoría denominada Documentación de la doctrina jurídica, que se caracteriza, en general, por ser una Documentación no tipificada, sin estructura definida, no controlada y con una vigencia ilimitada. Aunque con un lenguaje de léxico especial y propio de este tipo de información, la también llamada Bibliografía jurídica, entendida como cualquier estudio sobre temas jurídicos (en muchos casos se centran en el análisis de los textos legales y jurisprudenciales), sigue una difusión diferente al resto (repertorios de legislación y jurisprudencia, publicaciones oficiales...) a través de editoriales y revistas especializadas. Su publicidad y referencia se logran por medio de catálogos oficiales y privados. Integrada fundamentalmente por los tradicionales documentos bibliográficos en los que se publica la Literatura jurídica, independientemente del soporte o del formato, como son las monografías, los manuales 5 , los artículos de publicaciones periódicas, las actas de congresos y conferencias y la "literatura gris", goza de un alto nivel de publicación. Aunque la edición impresa juega un papel preponderante, las ediciones electrónicas (libros y revistas) se están integrando paulatinamente en la oferta de las principales editoriales jurídicas. Las bases de datos bibliográficas de carácter jurídico, por su concepción y finalidad, se asemejan al resto de bases de datos referenciales sin que quepa hacer diferenciación alguna en su forma de almacenamiento y utilización práctica. Reciben el nombre de bases de datos doctrinales (Páez Mañá, 1994, p.34) y recogen, de forma referencial, la doctrina jurídica publicada en libros, revistas, artículos de prensa, "literatura gris", etc...

Por todo lo dicho, se puede afirmar que la Documentación iusfilosófica es Documentación especializada en Ciencias Sociales y Humanidades, esencialmente Documentación jurídica que cuenta con las características propias de la Documentación

5 Según Maciá “la Bibliografía jurídica está constituida fundamentalmente por los manuales que se utilizan en las universidades para el estudio de la carrera de Derecho y por las monografías de investigación... que tienen su origen, en algunos casos, en tesis doctorales, en otros, de dictámenes encargados por alguna institución o colectivo" (Manual de documentación jurídica, 1998, p. 192). 
de la doctrina jurídica y que se ocupa de la información filosófico-jurídica contenida en cualquier soporte, considerando todos los usos de tal información, sobre todo en la enseñanza y la investigación, al tratarse de una disciplina (la Filosofía jurídica) eminentemente académica y universitaria que proporciona la perspectiva filosófica en torno al Derecho, y, por tanto, más abierta y amplia que la perspectiva dogmáticojurídica, la cual es propia de las demás ramas del Derecho. Su interdisciplinariedad y el uso de una tipología documental variada la hacen de gran interés para el estudioso de la Documentación.

\section{Conclusión: una reflexión interdisciplinar al respecto}

En primer lugar, debe destacarse la necesidad de la interdisciplinariedad entre las dos disciplinas planteadas, no sólo en la forma de afrontar el tema desde un plano teórico-conceptual sino también en relación con las prácticas concretas de los estudiosos de ambos campos, invitando a la necesaria cooperación entre sí para indagar en lo que caracteriza a cada una de las disciplinas académicas desde las perspectivas disciplinares recíprocas.

Desde este presupuesto general, podemos concluir que entre la Filosofía jurídica y la Documentación cabe establecer una relación de interdisciplinariedad de carácter lineal ${ }^{6}$ y bidireccional, ya que estamos ante la aplicación concreta de un determinado nivel de generalización de los conocimientos de una disciplina (la Documentación) en otra (la Filosofía jurídica) y viceversa.

En un primer sentido, tiene lugar una relación de carácter auxiliar de la Documentación con respecto a la Filosofía jurídica, puesto que la primera sirve de herramienta metodológica a la segunda en un momento muy concreto de su proceder epistemológico: el de la búsqueda y el tratamiento de la información que precisa para fundar materialmente sus investigaciones. Desde este punto de vista, el filósofo del Derecho tomará conciencia de la necesidad de su formación en técnicas de Documentación para un planteamiento y un desarrollo más sólidos de sus estudios.

Desde el otro lado de la interrelación, la Filosofía jurídica puede aportar a la Documentación interesantes elementos de juicio en lo que respecta, por ejemplo, al ámbito de la determinación y fijación de terminología documental especializada, proporcionando bases para la elaboración de tesauros, mapas conceptuales, y contribuciones a la conformación de arquitecturas de información en Centros de Documentación filosófica y jurídica. En este orden de ideas, puede constituir una valiosa contribución de cara a perfeccionar las prácticas documentales.

En definitiva, estamos ante el caso típico de relación interdisciplinar general que articula epistemológicamente la perspectiva de lo que se ha venido en llamar, desde

${ }^{6}$ Además de la lineal, se suele hablar de interdisciplinariedad estructural cuando la interrelación entre dos o más disciplinas o ramas del saber da lugar a marcos epistemológicos nuevos (ejemplos: la Bioquímica, la Biomecánica, etc....), y de interdisciplinariedad restringida, cuando la interrelación se produce exclusivamente en función de un objeto concreto. 
la Ciencia de la Documentación, como Documentación aplicada; y desde la Filosofía jurídica como Metodología de la investigación iusfilosófica.

\section{Referencias bibliográficas}

BUNGE, M. (1980). Epistemología. Barcelona: Ariel.

CARPINTERO BENÍTEZ, M. (2000). Historia breve del Derecho natural. Madrid: Colex.

COMTE, A. (1980). Discurso sobre el espíritu positivo, trad. de J. Marías. Madrid: Alianza Editorial.

GONZÁLEZ VICÉN, F. (1979a). "La Filosofía del Derecho como concepto histórico". En Estudios de Filosofía del Derecho. La Laguna: Universidad, Facultad de Derecho.

GONZÁLEZ VICÉN, F.(1979b). "El positivismo en la filosofía del derecho contemporánea". En Estudios de Filosofía del Derecho. La Laguna: Universidad, Facultad de Derecho.

HENKEL, H.(1968). Introducción a la Filosofía del Derecho: fundamentos del Derecho, trad. de E. Gimbernat Ordeig. Madrid: Taurus.

KUHN, S. (1971). La estructura de las revoluciones cientificas, trad. de A. Cotin. México: Fondo de Cultura Económica.

LEÓN HERNÁNDEZ, V. E. (2009). "La interdisciplinariedad: una concepción específica en el proceso docente educativo de la Física en la formación de técnicos agropecuarios”, en http://www.monografias.com/trabajos33/interdisciplinariedad/interdisciplinariedad.shtml, (fecha de consulta: 20 de julio de 2009).

LOPES GINEZ DE LARA, M.(2009). “Ciencias del lenguaje, Terminología y Ciencia de la Información: relaciones interdisciplinarias y transdisciplinariedad”, en http://dialnet. unirioja.es/servlet/fichero_articulo? codigo $=2532821$ \&orden $=0$, p. 4 , (fecha de consulta: 21 de julio de 2009).

LÓPEZ YEPES, J. (1995). La Documentación como disciplina. Teoría e historia. (2a ed. act. y amp.) Pamplona: EUNSA.

MANUAL de documentación jurídica. (1998). Edit. por Mateo Maciá. Madrid: Síntesis.

MÁRQUEZ GONZÁLEZ, J.A.(1985). Los enfoques actuales del Derecho natural. México: Porrúa.

MORIN, E. ( ) "Sur l'interdisciplinarieté". Bulletin du Centre International de Recherches et Études Transdisciplinaires (CIRET), n ${ }^{\circ} .2$.

PÁEZ MAÑÁ, J. (1994). Bases de datos jurídicos. Madrid: CINDOC, CSIC.

PÉREZ-ÁLVAREZ OSORIO, J.R. (1990). Introducción a la información y documentación cientifica. Madrid: Alambra.

POPPER, K. (1965). La lógica de la investigación cientifica, trad. de V. Sánchez de Zavala. Madrid: Tecnos.

RECASÉNS SICHES, L.(1970). Iusnaturalismos actuales comparados. Madrid: Facultad de Derecho, Universidad Complutense de Madrid.

ROBLES, G. (1988). Introducción a la Teoría del Derecho. Madrid: Debate.

ROMANOS DE TIRATEL, S. (2000) Guia de fuentes de información especializadas: Humanidades y Ciencias Sociales. 2a ed. Buenos Aires: GREBYD.

SERRANO VILLAFAÑE, E.(1977). Concepciones iusnaturalistas actuales (2a ed.) Madrid: Sección de Publicaciones de la Facultad de Derecho de la Universidad Complutense de Madrid. 
VILLASEÑOR RODRÍGUEZ, I. (2009). Fuentes de información en filosofía jurídica española (siglos XIX-XXI). Madrid: Dykinson.

VILLEY, M.(1984). Philosophie du Droit. Vol. II: "Les moyens du Droit" (2a ed.) Paris: Dalloz. 